Disclosure of Interests: None declared

DOI: 10.1136/annrheumdis-2020-eular.1556

\begin{tabular}{|l|l}
\hline FRI0180 & SURVEY ON HELP-SEEKING AND DISEASE \\
TRAJECTORY IN SYSTEMIC LUPUS ERYTHEMATOSUS
\end{tabular}

M. F. Eguez del Pozo ${ }^{1}$, M. Micelli ${ }^{1}$, A. Cañizares Calderón ${ }^{1}$, P. Avila ${ }^{1}$, N. Fara ${ }^{2}$ L. Garcia Faura ${ }^{3}$, D. Alvarez ${ }^{1}$, G. Sequeira ${ }^{1}$, E. Kerzberg ${ }^{1} .{ }^{1}$ Hospital J. M. Ramos Mejia, Servicio de Reumatologia, Ciudad Autonoma de Buenos Aires, Argentina; ${ }^{2}$ Clínica La Pequeña Familia, Junin, Argentina; ${ }^{3}$ Hospital Santa Francisca Romana, Arrecifes, Argentina

Background: Few studies in Latin America have analysed the time lag since patients experience the first joint symptoms until they consult a physician, and a diagnosis is made, and most of them have dealt with patients sustaining Rheumatoid Arthritis (RA) ${ }^{1-2}$.

Objectives: To study both patient and disease factors that have some bearing on the time lag until a physician is first consulted and a diagnosis is made.

Methods: Multiple-choice survey to patients of 18 years of age or older who met Systemic Lupus Erythematosus (SLE) Classification Criteria (2012). The following aspects were measured: time lag from symptom onset to first medical consultation (Time 1), time lag since first medical consultation until a diagnosis is made (Time 2), and time lag until the first consultation with a rheumatologist.

Patients were interviewed at the time or within 6 months of diagnosis, and asked about the physician they first consulted and the one who made the diagnosis. They were also requested to describe one disease onset out of 4 trajectories: acute, acute with temporary remissions, chronic with partia improvements, or sustained chronic. Patients were required to recount their response to the first symptoms, the limitations brought about by the disease (employing the Steinbrocker scale), and whether help-seeking was first aimed at consulting a physician or they decided to wait. A group of patients with Rheumatoid Arthritis (RA) answered the same survey. Every patien signed an informed consent form, and the study was approved by the Hospital Bioethics Committee.

Results: Twenty-eight patients with SLE and 29 patients with RA filled in the survey. SLE patients were younger as expected (32 years vs 49 years; $p<0.001$ ) Acute was the most common disease trajectory in SLE patients (36\% vs $34 \%$; $p$ 0.8 ) and chronic in RA (38\% vs $32 \% ; p$ 0.8). The first professional consulted was an on-duty physician in SLE ( $36 \%$ vs $24 \%$ in RA; $p$ 0.5). Except for the matter of computer at home and/or a mobile phone with an Internet connection at home, both groups were similar, although SLE patients showed a trend to better leve education and lesser impact of the disease on daily life at the beginning (see Table 1).

Table 1.

\begin{tabular}{lccc}
\hline & SLE & RA & P \\
\hline $\mathrm{n}$ & 28 & 29 & \\
Education (median) & Secondary & Secondary & 0.07 \\
Had a job & $11(39 \%)$ & $15(52 \%)$ & 0.5 \\
Had health insurance & $8(29 \%)$ & $15(52 \%)$ & 0.1 \\
Had computer/mobile phone with an Internet connection & $25(89 \%)$ & $18(62) \%$ & 0.04 \\
Steinbrocker (median with range 25-75\%) & 3 & 3 & 0.06 \\
Immediately sought medical help & $(2-3)$ & $(3-4)$ & \\
Did not know what to do when the first symptoms appeared & $15(54 \%)$ & $16(56 \%)$ & 0.9 \\
Diagnosis made by a rheumatologist & $7(25 \%)$ & $13(45 \%)$ & 0.2 \\
& $20(71 \%)$ & $23(80 \%)$ & 0.7 \\
\hline
\end{tabular}

Time 1 in patients with SLE was significantly lower with a median of 7 days range (25-75\%) 1-30 days vs 30 days in RA, range 14-180 ( $p 0.01$ ), and the former also showed a trend to lower median Time 2: 81 days, range 25-209 vs 185 days in RA, range 60-275 (p 0.1). Besides, they showed a significantly shorter time lag until the first visit to a rheumatologist with a median of 120 days, range $35-225$ vs 330 days, range $120-450$ in patients with RA (p 0.005)

Conclusion: SLE patients consult a physician and visit a rheumatologist sooner than RA patients do, and they also show a trend to obtain a faster diagnosis. Education, health insurance, employment, the disease impact on their daily life and initial response to the symptoms do not appear to account for their swiftness in seeking medical help.

References:

[1] Mussano E, Missakian S, Onetti CM (2007) Estudio demográficosocial de pacientes con artritis reumatoidea en la provincia de Córdoba (Argentina). Rev Argent Reumatol 4:13-22.

[2] Rodríguez-Polanco E, Al Snih S, Kuo YF et al (2011) Lag time between onset of symptoms and diagnosis in Venezuelan patients with rheumatoid arthritis. Rheumatol Int 31:657-665. doi:10.1007/s00296-009-1358-9.
Disclosure of Interests: None declared

DOI: 10.1136/annrheumdis-2020-eular.2668

\section{FRI0181 \\ ORGAN DAMAGE FREE SURVIVAL IN SOUTHERN CHINESE PATIENTS WITH ACTIVE LUPUS NEPHRITIS}

C. C. Mok ${ }^{1}$, C. S. $\operatorname{Sin}^{1}$, K. C. Hau' ${ }^{1}$ T. H. Kwan ${ }^{1}$ on behalf of NA. ${ }^{1}$ Tuen Mun Hospital, Medicine, Hong Kong, Hong Kong (SAR)

Background: The goals of treatment of lupus nephritis (LN) are to induce remission, retard the progression of chronic kidney disease, prevent organ complications and ultimately reduce mortality. Previous cohort studies of LN have mainly focused on the risk of mortality and development of end stage renal failure (ESRF) (renal survival). The cumulative frequency of LN patients who survive without organ damage, which correlates better with the balance between treatment efficacy and toxicity, as well as quality of life, has not been well studied.

Objectives: To study the organ damage free survival and its predictive factors in patients with active LN.

Methods: Consecutive patients who fulfilled $\geq 4$ ACR/SLICC criteria for SLE and with biopsy proven active LN between 2003 and 2018 were retrospectivey analyzed. Those with organ damage before LN onset were excluded. Data on renal parameters and treatment regimens were collected. Complete rena response (CR) was defined as normalization of serum creatinine (SCr), urine $\mathrm{P} / \mathrm{Cr}$ (UPCR) $<0.5$ and inactive urinary sediments. Partial renal response (PR) was defined as $\geq 50 \%$ reduction in UPCR and $<25 \%$ increase in SCr. Organ damage of SLE was assessed by the ACR/SLICC damage index (SDI). The cumulative risk of having any organ damage or mortality since LN was studied by Kaplan-Meier's analysis. Factors associated with a poor outcome were studied by a forward stepwise Cox regression model, with entry of covariates with $p<0.05$ and removal with $p>0.10$.

Results: 273 LN patients were identified but 64 were excluded (organ damage before LN onset). 211 LN patients were studied (92\% women; age at SLE $30.4 \pm 13.5$ years; SLE duration at LN 1.9 \pm 3.1 years). $47(22 \%)$ patients had nephrotic syndrome and $60(29 \%)$ were hypertensive. Histological LN classes was: III/IV $\pm \mathrm{V}(75.1 \%), \mathrm{I} / \mathrm{II}(7.8 \%)$ and pure $\mathrm{V}(17.1 \%)$ (histologic activity and chronicity score $7.0 \pm 4.2$ and $1.8 \pm 1.5$, respectively). Induction regimens were: prednisolone $(33.1 \pm 17.5 \mathrm{mg} /$ day) in combination with intravenous cyclophosphamide (CYC) $(21.4 \% ; 1.0 \pm 0.2 \mathrm{~g}$ per pulse), oral CYC $(8.6 \% ; 96.4 \pm 37.8 \mathrm{mg} / \mathrm{day})$, azathioprine (AZA) (14.3\%; 78.6 $\pm 25.2 \mathrm{mg} /$ day), mycophenolate mofetil (MMF) $(22.8 \% ; 1.9 \pm 0.43 \mathrm{~g} /$ day) and tacrolimus (TAC) $(17.1 \% ; 4.3 \pm 1.1 \mathrm{mg} /$ day). After a follow-up of $8.6 \pm 5.4$ years, $94(45 \%)$ patient developed organ damage (SDI $\geq 1$ ) and $21(10 \%)$ patients died. The commonest organ damage was renal $(36.3 \%)$ and musculoskeletal (17.9\%), and the causes of death were: infection $(38.1 \%)$ malignancy (19.0\%), cardiovascular events $(9.5 \%)$ and ESRF complications $(9.5 \%)$. At last visit, 114 (55\%) patients survived without any organ damage. The cumulative organ damage free survival at 5,10 and 15 years after renal biopsy was $73.5 \%, 59.6 \%$ and $48.3 \%$, respectively. The 5, 10 and 15 -year renal survival rate were $95.2 \%, 92.0 \%$ and $84.1 \%$ respectively. In a Cox regression model nephritic relapse (HR 3.72[1.78-7.77]), proteinuric relapse (HR 2.30[1.07-4.95]) and older age (HR 1.89[1.05-3.37]) were associated with either organ damage or mortality, whereas CR (HR 0.25[0.12-0.50]) at month 12 were associated with organ damage free survival. Baseline SCr, UPCR and histological LN classes were not significantly associated with a poor outcome. Among patients with class III/IV LN, the long-term organ damage free survival were not significantly different in users of MMF (reference) from CYC (IV/oral) (HR 1.45[0.76- 2.75]) or TAC (HR $1.03[0.26-1.62])$ as induction therapy.

Conclusion: Organ damage free survival is achieved in $55 \%$ of patients with active LN upon 9 years of follow-up. CYC/MMF/TAC based induction regimens did not differ for the long-term outcome of LN. Targeting complete renal response and preventing renal relapses remain important goals of $L N$ treatment.

Acknowledgments: NIL

Disclosure of Interests: None declared

DOI: 10.1136/annrheumdis-2020-eular.2552

\section{FRI0182 RISK FACTORS ASSOCIATED WITH RENAL INVOLVEMENT IN PRIMARY SJÖGREN'S SYNDROME: DATA FROM THE SPANISH SJÖGRENSER COHORT}

J. Narváez ${ }^{1}$, C. Sánchez-Piedra ${ }^{2}$, M. Fernandez Castro ${ }^{3}$, V. Martinez Taboada $^{4}$, A. Olive ${ }^{5}$, J. Rosas ${ }^{6}$, A. García-Vadillo ${ }^{7}$, E. Judez ${ }^{8}$, E. Ruiz Lucea ${ }^{9}$, L. Romani ${ }^{10}$, J. L. Andreu ${ }^{3}$ on behalf of SJONGRENSER Project. ${ }^{1}$ Hospital Universitario de Bellvitge, Rheumatology, Barcelona, Spain; ${ }^{2}$ Unidad de Investigación de la Sociedad Española de Reumatologia, Madrid, Spain; ${ }^{3}$ Hospital Puerta de Hierro, Rheumatology, Madrid, Spain; ${ }^{4} \mathrm{Hospital}$ Marqués de Valdecilla, Rheumatology, Santander, Spain; ${ }^{5}$ Hospital Germans Trias i Pujol, Rheumatology, Barcelona, Spain; ${ }^{6}$ Hospital Marina Baixa, Rheumatology, Alicante, Spain; ${ }^{7}$ Hospital de la Princesa, Rheumatology, 
Madrid, Spain; ${ }^{8}$ Hospital de Albacete, Rheumatology, Albacete, Spain; ${ }^{9}$ Hospital de Basurto, Bilbao, Spain; ${ }^{10}$ Hospital Virgen de las Nieves, Rheumatology, Granada, Spain

Objectives: To investigate the prevalence, risk factors, and effects of primary renal disease on morbidity and mortality in patients with primary Sjögren's syndrome (pSS).

Methods: All patients in the SJÖGRENSER (registry of adult SSp patients of the Spanish Society of Rheumatology, cross-sectional phase) cohort were retrospectively investigated for the presence of clinically significant renal involvement directly related to $\mathrm{pSS}$ activity.

Results: Of the 437 patients investigated, 39 (9\%) presented overt renal involvement during follow-up. Severe renal disease necessitating kidney biopsy was relatively rare $(23 \%)$.

Renal involvement may complicate pSS at any time during the disease course and is associated with severe disease (indicated by higher scores of involvement, activity, and damage), systemic multiorgan involvement, and a higher frequency of lymphoma. Multivariate analysis showed that older age (odds ratio [OR] 1.03, 95\% confidence interval [Cl] 1.00-1.07), higher European League Against Rheumatism Sjogren's Syndrome Disease Activity Index scores (OR 1.1, $\mathrm{Cl} 1.03-1.18$ ), serum anti-La/SSB positivity (OR 6.44, $\mathrm{Cl} 1.36-30.37$ ), and non-vasculitic cutaneous involvement (OR 8.64, 1.33-55.90) were independently associated with this complication.

Chronic renal failure developed in 23 of 39 patients (59\%); only 1 of them progressed to end-stage renal disease necessitating renal replacement therapy. Patients with renal disease showed higher Sjögren's syndrome disease damage index scores (SSDDI), higher rates of hospitalization due to disease activity and higher rates of clinically relevant comorbidities.

Conclusion: Renal involvement is an uncommon complication in pSS that was observed in $9 \%$ of patients. Although categorized as a non-negligible comorbidity, this condition shows a favorable prognosis.

Disclosure of Interests: None declared

DOI: 10.1136/annrheumdis-2020-eular.5149

\section{FRI0183 DISTINCTIVE TRAITS OF MYOCARDIAL INFLAMMATION IN PATIENTS WITH SYSTEMIC LUPUS ERYTHEMATOSUS: A MULTICENTRE STUDY}

G. A. Ramirez ${ }^{1,2}$, M. Gerosa ${ }^{3,4}$, G. De Luca ${ }^{1,2}$, L. Beretta ${ }^{5}$, S. Sala ${ }^{6}$, G. Peretto ${ }^{6}$, L. Moroni ${ }^{1,2}$, F. Mastropaolo ${ }^{1,2}$, A. Cariddi ${ }^{1}$, S. Sartorelli ${ }^{1}$, C. Campochiaro ${ }^{1,2}$, E. Bozzolo ${ }^{1}$, R. Caporali ${ }^{3,4}$, L. Dagna ${ }^{1,2}$ on behalf of Milan SLE Consortium (SMiLE). ${ }^{1}$ IRCCS H San Raffaele, UnIRAR, Milan, Italy; ${ }^{2}$ Università Vita-Salute San Raffaele, Milan, Italy; ${ }^{3}$ ASST Gaetano Pini -CTO, Clin. Rheumatology Unit, Milan, Italy; ${ }^{4}$ University of Milan, Dep. of Clin. Science of Community Health, Milan, Italy; ${ }^{5}$ Fondazione IRCCS Ca' Granda H Maggiore Policlinico, Referral Center for Systemic Autoimmune Diseases, Milan, Italy; ${ }^{6}$ RCCS H San Raffaele, Unit of Cardiology, Milan, Italy

Background: Myocarditis is an infrequent but potentially life-threatening inflammatory disorder and might be part of the spectrum of systemic lupus erythematosus (SLE). Little is known about the clinical and histologic features of myocarditis in SLE, especially compared to other forms of myocarditis.

Objectives: to test for potential distinctive traits among myocarditis in SLE (MyoSLE), SLE without myocarditis (OnlySLE) and myocarditis without SLE (OnlyMyo)

Methods: Patients with MyoSLE were identified from three centres and compared with 231 cross-sectionally enrolled patients with OnlySLE and 87 patients with OnlyMyo. MyoSLE patients were split into two groups based on myocarditis onset within (early onset) vs after (late onset) the first year from SLE diagnosis. OnlySLE patients were dichotomised in the same way based on disease duration at time of enrolment. Demographics and general clinical features were collected retrospectively. SLE disease activity index 2000 (SLEDAI-2K), SLE International Collaborating Clinics/American College of Rheumatology damage index (SDI), clinical and laboratory features were collected at time of myocarditis onset in MyoSLE and at enrolment in OnlySLE. Quantitative data are expressed as median [interquartile range].

Results: Fourteen MyoSLE patients were identified, $50 \%$ with early onset. Women were equally frequent among MyoSLE (71\%) and OnlySLE patients $(87 \%)$ and less frequent in the OnlyMyo group (43\%; $p<0.001)$. Age was comparable among groups. Clinical features at presentation, including left ventricular ejection fraction, were similar between MyoSLE and OnlyMyo, although the former had higher levels of pro-brain natriuretic peptide (1.1 [0.4-1.8] vs 0.1 [0.1$0.5] \mathrm{ng} / \mathrm{ml} ; \mathrm{p}=0.004)$. Patients with MyoSLE also had a lower frequency of left ventricle lateral wall involvement ( 36 vs $68 \% ; p=0.035$ ) and of oedema (20 vs $71 \% ; p=0.036$ ) and necrosis (0 vs $64 \% ; p=0.009$ ) at biopsy. Antiphospholipid antibodies (aPL) were more frequent in MyoSLE (57\%) compared to both OnlyMyo $(16 \% ; p=0.003)$ and OnlySLE $(28 \% ; p=0.031)$. Compared to OnlySLE, patients with MyoSLE also had a higher prevalence of aPL-syndrome (APS: 36 vs $7 \% ; p=0.003$ ), neuropsychiatric (NPSLE: 43 vs $19 \% ; p=0.039$ ) and gastrointestinal manifestations ( $21 \mathrm{vs} 5 \% ; p=0.045$ ). Early and late onset patients had similar demographics and clinical features and did not differ from patients with OnlySLE with similar disease duration in terms of SLEDAI-2K and SDI. Late onset MyoSLE patients had a higher prevalence of NPSLE (57 vs $18 \%$; $p=0.026)$ and APS ( 57 vs $7 \% ; p=0.001)$ and higher $C$-reactive protein levels ( 6 [2-12] vs $1[0-4] \mathrm{mg} / \mathrm{l} ; \mathrm{p}=0.024)$ compared to OnlySLE patients with the same disease duration.

Conclusion: Demographics of patients with MyoSLE are more similar to patients with OnlySLE than to OnlyMyo patients. MyoSLE might have distinct histological and pathogenic features compared to OnlyMyo. Patients with MyoSLE show similar patterns of disease activity and accrued damage at time of myocarditis onset compared to patients with OnlySLE with the same disease duration but might diverge later on in SLE course. aPL are frequent in MyoSLE and might both contribute to the pathogenesis of myocardial inflam mation and account for the high prevalence of NPSLE and APS, especially in late onset cases.

\section{References:}

[1] Gartshteyn $Y$ et al., Lupus, 2020

[2] Thomas G et al., J Rheumatol, 2017

[3] Peretto G et al., Int J Cardiol, 2019

[4] McDonnell T et al., Blood Rev, 2019

Disclosure of Interests: Giuseppe Alvise Ramirez: None declared, Maria Gerosa: None declared, Giacomo De Luca Speakers bureau: SOBI, Novartis Celgene, Pfizer, MSD, Lorenzo Beretta Grant/research support from: Pfizer, Simone Sala: None declared, Giovanni Peretto: None declared, Luca Moroni: None declared, Francesca Mastropaolo: None declared, adriana cariddi: None declared, Silvia Sartorelli: None declared, Corrado Campochiaro Speakers bureau: Novartis, Pfizer, Roche, GSK, SOBI, Enrica Bozzolo: None declared, Roberto Caporali Consultant of: AbbVie; Gilead Sciences, Inc.; Lilly; Merck Sharp \& Dohme; Celgene; Bristol-Myers Squibb; Pfizer; UCB, Speakers bureau: Abbvie; Bristol-Myers Squibb; Celgene; Lilly; Gilead Sciences, Inc; MSD; Pfizer; Roche; UCB, Lorenzo Dagna Grant/research support from: The Unit of Immunology, Rheumatology, Allergy and Rare Diseases (UnIRAR) received unresctricted research/educational grants from Abbvie, Bristol-Myers Squibb, Celgene, Janssen, Merk Sharp \& Dohme, Mundipharma Pharmaceuticals, Novartis, Pfizer, Roche, Sanofi-Genzyme, and SOBI., Consultant of: Prof Lorenzo Dagna received consultation honoraria from Abbvie, Amgen Biogen, Bristol-Myers Squibb, Celltrion, Novartis, Pfizer, Roche, Sanofi-Genzyme, and SOBI.

DOI: 10.1136/annrheumdis-2020-eular.4653

\section{FRI0184 \\ ATTRIBUTION OF NEUROPSYCHIATRIC MANIFESTATIONS TO SYSTEMIC LUPUS ERYTHEMATOSUS IN POLISH COHORT OF PATIENTS WITH THE USE OF THE ITALIAN MODEL}

K. Pawlak-Bus ${ }^{1,2}$, W. Schmidt ${ }^{1,2}$, P. Leszczynski ${ }^{1,2} .{ }^{1}$ Poznan University of Medical Sciences, Department of Rheumatology and Rehabilitation, Poznan, Poland; ' Jozef Strus Municipal Hospital, Department of Rheumatology and Osteoporosis, Poznan, Poland

Background: Distinguishing primary NPSLE (neuropsychiatric systemic lupus erythematosus) from secondary causes remains challenging (1). Attribution models were developed in order to aim clinicians in correct classification of NPSLE cases (2).

Objectives: To investigate the prevalence of primary NPSLE manifestations assigned with Italian model of attribution (2).

Methods: We retrospectively assessed clinical details of 164 patients with SLE classified with 2012 SLICC (Systemic Lupus International Collaborating Clinics) classification criteria, 21 were excluded due to incomplete information. Data was gathered with a questionnaire comprising demographics, medical history, laboratory results (concentrations of antibodies against double stranded DNA - anti-dsDNA, complement components C3 and C4), disease activity measured with Systemic Lupus Erythematosus Disease Activity Index 2000 (SLEDAI-2K) and Physician Global Assessment (PGA) and damage determined with SLICC/ ACR (American College of Rheumatology) Damage Index (SDI). Neuropsychiatric manifestations were categorized in accordance with 1999 ACR glossary and attribution of manifestations was performed with the use of Italian model with the score $\geq 7$ out of 10 points enabling assignment to primary NPSLE group (2). Statistical analysis was conducted with Statistica v.13.3 using Mann-Whitney U, chi-square and Fisher exact test.

Results: We encountered 155 NP manifestations in our cohort and 52 (34\%) were attributed to SLE. Characteristics of the study groups are presented in Table 1. Exact manifestations and their attribution rates are presented on 